\title{
Kmax-based Event Mode Data Acquisition System for the University of Kentucky Accelerator Laboratory
}

\author{
Benjamin P. Crider ${ }^{* \dagger}$ \\ Department of Physics and Astronomy, University of Kentucky, Lexington, KY 40506-0055 USA \\ E-mail: ben.crider@uky.edu \\ Rodney B. Piercey \\ Department of Physics and Astronomy, Eastern Kentucky University, Richmond, KY 40475 USA \\ E-mail: rodney.pierceyeeku.edu
}

\begin{abstract}
The University of Kentucky Acceleratory Laboratory (UKAL), a facility that possesses unique experimental capabilities for the scattering and detection of monoenergetic fast neutrons, has recently invested in updating its data acquisition capabilities. Starting with a new system for high-precision singles measurements, where subsequent analysis leads to the extraction of lifetimes in the femtosecond region via the Doppler-shift attenuation method, we have developed an event mode coincidence data acquisition system with capabilities that allow for a new range of experiments to be performed.

The Kmax-based Event Mode Data Acquisition System at UKAL utilizes existing CAMAC modules and crates, as well as the Wiener CC-USB CAMAC controller. Developed with Sparrow Corporation's Kmax 10, utilizing Java 7 and widget-based toolsheets, the system allows for high throughput with low dead times. Currently, the system is capable of supporting 40 ADCs, 32channels of TDC, 12-channels of scaler values, and 1 Mword of in-crate memory buffering. In this presentation, the commissioning, operation, and performance of the system will be described, and the particular design choices used for the graphical user interface and the logical flow of data acquisition and translation will be discussed.
\end{abstract}

Technology and Instrumentation in Particle Physics 2014,

2-6 June, 2014

Amsterdam, the Netherlands

*B.P.C. thanks his dissertation advisor, Prof. Steven W. Yates, for support and funding for hardware, software, and time needed to complete this project.

† current address: National Superconducting Cyclotron Laboratory, East Lansing, MI 48824-1321. current email: crider@nscl.msu.edu

${ }^{\ddagger}$ Speaker. 


\section{Introduction}

For many decades now, careful analysis of nuclear spectra has involved digitizing signals from detectors either near the end of the pulse processing with analog acquisition systems or, more recently, shortly after detection with digital acquisition systems. The data acquisition systems are tasked with the job of controlling how and when the data is moved to high-capacity storage devices on a computer. Additionally, a data acquisition system typically must be able to offer an online display on data as it is being taken, giving users information about the status of their experiment as well as allowing for identification of problems with the experimental setup.

The tools for creating data acquisition systems have been improving rapidly over recent years. Today, there are now many software systems upon which users can build powerful, user-friendly acquisition systems that use high-level programming languages and allow for excellent graphical interfaces. One such system is Kmax 10 [1], a complete environment for data acquisition and instrument control built on a Java 7 framework. UKAL has recently developed a new, event mode data acquisition system using Kmax in order to update the previous acquisition system as well as expand the experimental capabilities of the lab.

\section{Motivation}

The previous event mode data acquisition system at UKAL was an in-house-developed graphical acquisition system originally designed for Windows 95 and then upgraded to use Linux kernel 2.4 and object-oriented programming $\left(\mathrm{C}^{++}\right)$in 2005. Communication between the computer and the CAMAC crate was achieved using a DSP Model 6001 CAMAC Crate Controller. While adequate for its time, the DSP Model 6001 was a limiting factor by today's standards. These limitations included 8-bit data transfers of 24-bit data words, transfer speeds of only $900 \mathrm{KBytes}$ per second, and a 40-pin connector for interfacing the module to a computer, which is now defunct and can only be found on specially ordered motherboards in current computers. The hardware restrictions imposed by this device prevented a computer upgrade for several years.

Further motivation for a new data acquisition system was the old system's graphical display; the low-resolution display of single-colored dots on a black background made it difficult to view fine structures in the online spectra. Additionally, only a single spectrum could be viewed at any given time and moving through different regions of the spectrum was a cumbersome process of cycling through predefined ranges without allowing for placing cuts on matrices and viewing their projections. The definition of parameters within the program was also a frequent source of confusion. If defined improperly, a seemingly innocuous mistake could have disastrous effects on the outcome of the spectra.

Perhaps the greatest restriction was the hard-wired CAMAC crate configuration that was suited for only a few modules, such that any changes to the modules used would require significant changes to the code itself. In particular, the lack of support for in-crate buffering severely limited the throughput of the system. By requiring the crate to be serviced and read out upon every event, count rates of only a few thousand counts per second could result in nearly $90 \%$ dead time.

An upgrade to the data acquisition system was required in order to alleviate these issues and allow for the use of more modern computer hardware and storage capabilities. However, budget 
constraints required that the upgrade to the system use the crates and hardware that UKAL already owned, which were all CAMAC. Additionally, the majority of the work load was to be performed by a single graduate student, meaning that building a system from the ground up was far less attractive than using software that already had many tools for instrument control and data visualization. In the end, Kmax met both of these requirements.

\section{Kmax-Based Event Mode Data Acquisition System}

Development of the Kmax-based event mode data system has been completed and utilized in a commissioning run (see Section 4). The system exists as a Kmax 10 toolsheet with that contains all graphical widgets associated with the user interface and all non-proprietary Java 7 code related to both the acquisition processes as well as the user interaction with the GUI widgets. The acquisition system was designed to use the Wiener CC-USB CAMAC Controller [2], which supports full 24bit data transfers and USB 2.0 data transfer rates (480 Mbit/s). This improvements allow for an immediate decrease in the dead-time introduced from transferring data from the hardware modules to the computer.

Undoubtedly, the largest gain in dead-time reduction comes from implementing the Cheescote Mountain CAMAC (CMC) 203 FERA Driver, Memory and Histogrammer module. The gains of using the CMC203 are two-fold; a 1 Mword buffer allows for in-crate buffering such that every gate triggered event does not have to be read to the computer and the data transfers occur along the FERAbus, allowing them to proceed at 100-ns-per-word rates. This increased data transfer rate (faster than USB 2.0 speeds) and the ability to buffer data in the crate so that a large, block transfer of data can occur periodically were key in allowing for low dead times at high input rates.

What follows are some of the key features of the system.

\subsection{CAMAC Configuration GUI}

The acquisition system is currently capable of supporting a single CAMAC crate of modules. The modules capable of being used are 1 LeCroy 337732 Channel CAMAC TDC, up to 10 of Ortec AD413 4 Channel 8K ADCs and Ortec AD114 16K ADCs in any combination, 1 Lecroy 255112 Channel Scaler, 1 Cheesecote Mountain CMC203 FERA Driver, and 1 Wiener CC-USB CAMAC controller. For each of the ADCs, TDC, and scaler, a GUI has been provided where the user can select which modules and channels are being used, as shown in Figure 1. This allows for a userfriendly setup process, as all parameter calculations and assignments are performed automatically based on the modules selected in this GUI.

\subsection{2-D Histograms}

Online 2-D histograms can be defined to contain any parameter based on the signal inputs to the system, be it energy, time of flight, pulse-shape discrimination, etc. Due to the large amount of memory used by each 2-D histogram and the design choice to keep all data from a run present in each 2-D histogram, a preset number of histograms are placed on the toolsheet such that, in the current version of the system, a maximum of 18 2-D histograms may be defined. More can be added by copying the current widgets and modifying the code in a few places to account for the extra histograms. There are two unique displays, as shown in Figures 2 and 3. Each display has a 


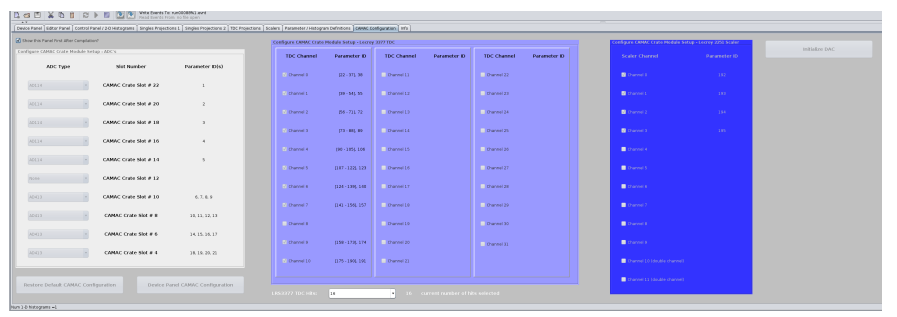

Figure 1: The two screens that supply the GUI for defining the modules and their channels used for an experiment.

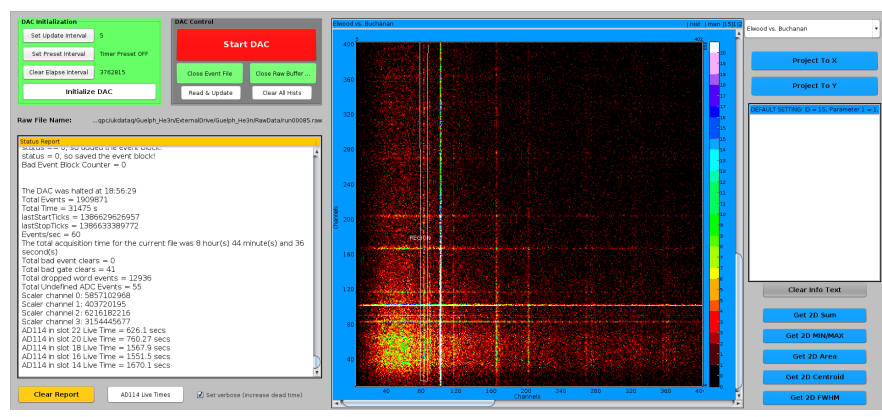

Figure 2: Left screen of the 2-D Histogram and control panel.

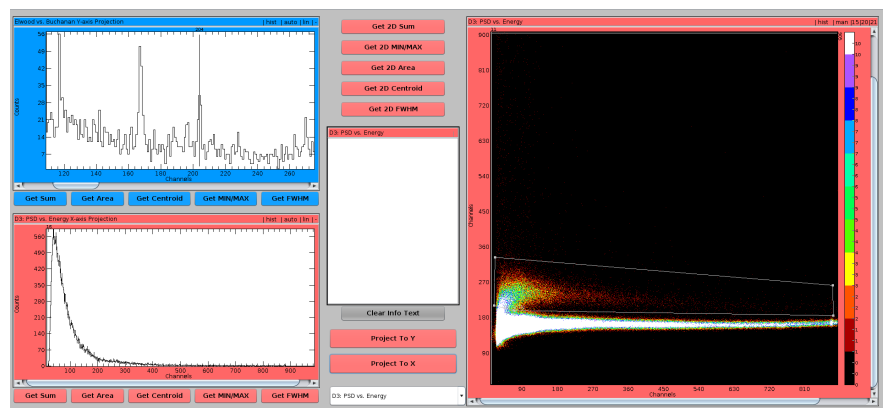

Figure 3: Right screen of the 2-D Histogram and control panel.

region that can be moved to any point on the histogram by clicking and dragging its vertices (where more vertices can be added for more complex shapes). Within this region, the ability to determine 2-D areas, sums, centroids, full-width-at-half-maximum (FWHM), and $\min / \mathrm{max}$ have been given, as well as the ability to project the data onto either the $\mathrm{x}$ - or $\mathrm{y}$-axis. A combo box has been given to allow the user to view any of the predefined 2-D histograms at any time.

\subsection{1-D Histograms}

1-D histograms for each defined parameter can be viewed in addition to the predefined 2-D histograms. While not a memory issue, the large amount of screen space required to put 1-D histograms on the screen means that currently only $241-\mathrm{D}$ histograms, though this is once again expandable by copying widgets and modifying code in a few places. The user again has the ability to determine sums, areas, centroids, FWHM, and $\min / \max$ between a pair of cursors. Figure 4 shows one of the 1-D Histogram display screens. 


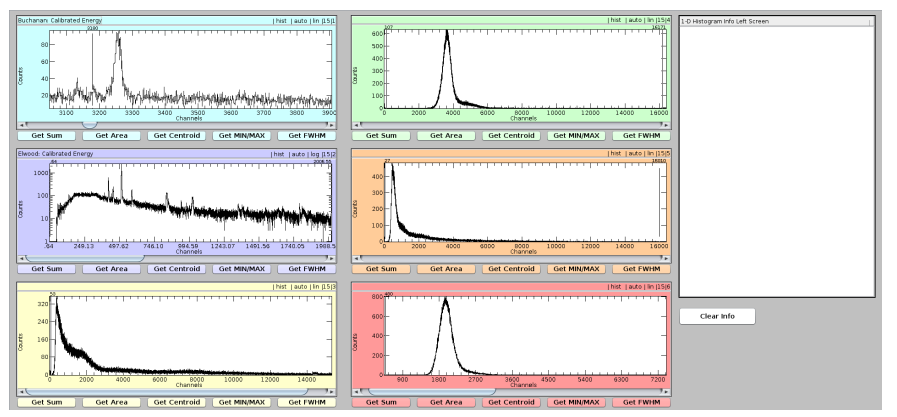

Figure 4: One of the four screens that display online, 1-D histograms of the defined parameters.

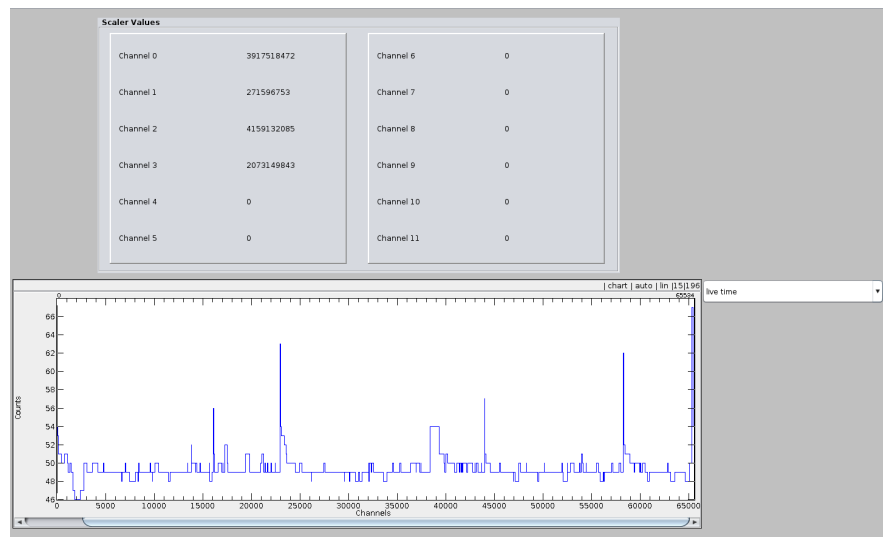

Figure 5: One of the two screens that supply online, scaler value reporting and strip charts. Derived quantities, such as system dead time, can also be displayed.

\subsection{Scalers}

Scaler information is recorded using the LeCroy 255112 Channel Scaler. However, this module does not connect along the FERAbus like all other modules. Thus, there is no associated time ordering with the scaler counts. This is managed by recording the scaler values and adding their values to the end of each data event during a readout. Thus, when strip charting these values, the width of the scaler "event" is proportional to the total number of event triggers received between readouts.

Derived quantities, such as system dead time, are calculated as ratios of scaler inputs and are capable of being displayed as a strip chart as well. This gives users a visual representation of such quantities, allowing for immediate identification of issues related to them. Figure 5 shows how the scaler values, as well as one of the strip charts, are displayed within the system.

\subsection{TDC}

The implementation of the Lecroy 337732 channel TDC allows for a wide range of input time differences (up to $32 \mu \mathrm{s}$ ) as well as the ability to record up to 16 hits per event. In utilizing these features, the TDC spectrum for each channel ends up being a derived parameter which is a sum of the individual hits, which also each have their own parameter. Thus, the online view already accounts for the total hits in each TDC channel. Other derived quantities capable of being 


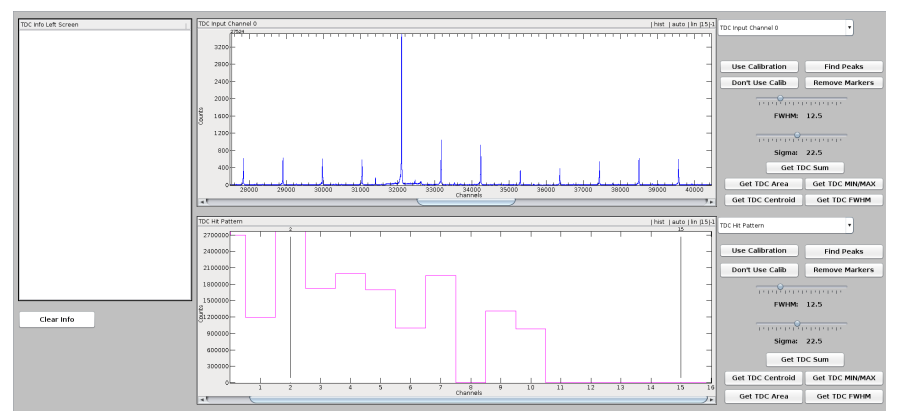

Figure 6: One of the two screens that supply online, TDC histograms. Derived quantities, such as system hit patterns, can also be displayed.

displayed are the hit pattern, which shows the number of hits in each TDC channel against the channel number and allowing for comparison between the detectors used as input, as well as hit multiplicity spectrum, which shows the number of hits (up to 16) each detector is receiving in any given event. Figure 6 shows how half of the TDC online spectra are displayed within the system.

\subsection{Raw Data Translation}

The logic behind sorting the raw data into events was modularized in the code to match the unique characteristics of the data words originating from a particular module in the CAMAC crate. Each piece of data from a module is accompanied by a header word, allowing for association of that data word with the module that sent it. The CMC203 FERA Driver inputs a "gate header" at the beginning of each event and a "clear" header at the end of each event. In addition to signaling the beginning and end of an event, these words also supply information on the status of the system during the event, allowing the algorithm to know whether or not it should keep the event.

While traversing the raw data buffer, the algorithm uses the calculated parameters based on the crate configuration (see Section 3.1) and assigns data from each module to the appropriate parameter. If there is an issue with the ordering of the data words or the number of data words that should be present after a particular header word, the system will drop the current event and all subsequent events until a valid "gate header" is found, at which point the algorithm is "re-synced" and can begin sorting once again. Figure 7 shows a flow chart of how sorting a good event proceeds within the algorithm.

\section{Commissioning Run}

A commissioning run using the Kmax-based event mode data acquisition system was performed at UKAL using 8 deuterated benzene scintillators (designed for the DESCANT array at TRIUMF [4]) for neutron detection and 2 high purity germanium (HPGe) detectors for $\gamma$ ray detection in a ${ }^{24} \mathrm{Mg}\left({ }^{3} \mathrm{He}, \mathrm{n}\right){ }^{26} \mathrm{Si}$ experiment that utilized UKAL's capabilities for monoenergetic neutron production. The detector setup is shown in Figure 8.

Setup for the experiment resulted in the definition of 195 parameters per event, which includes all inputs to ADCs, the TDC (including multiple hits), the scaler, and any derived quantities. Thus, upon each readout all raw data was sorted into events of these 195 parameters and all defined 


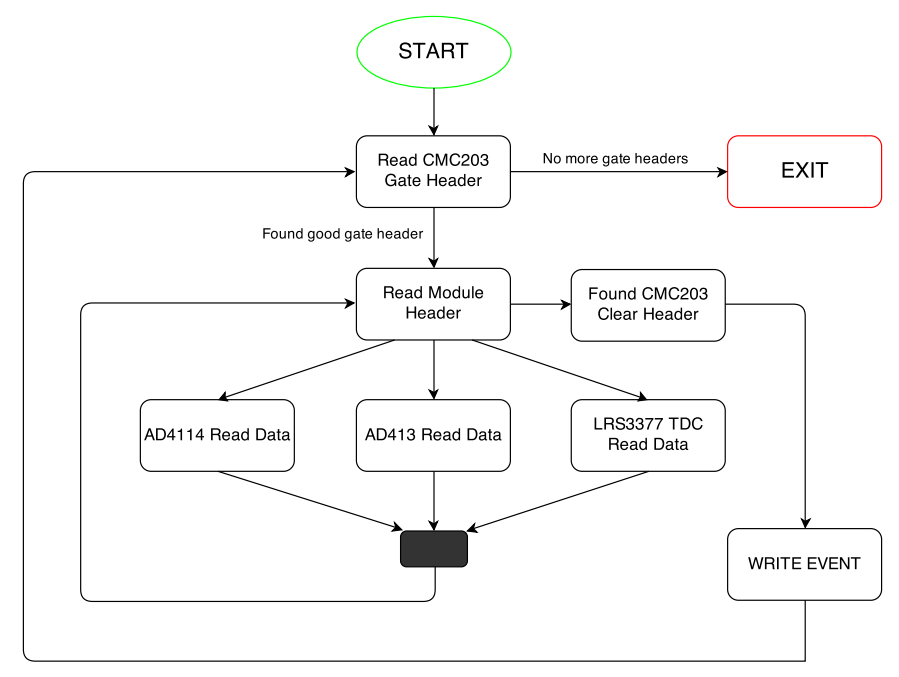

Figure 7: Flow chart of the logic behind translating the raw data into sorted events. The error handling for dropped or improperly organized words is not shown.

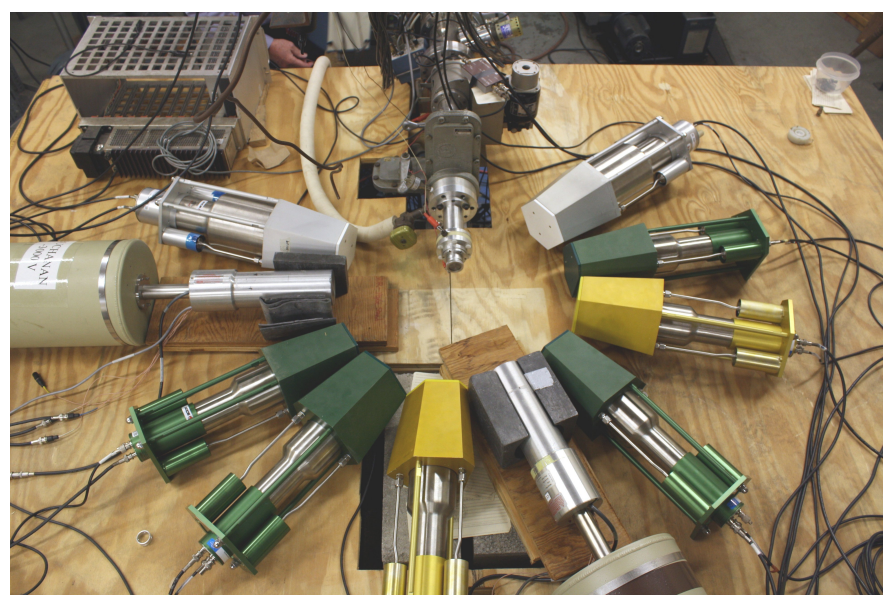

Figure 8: Experimental setup for ${ }^{24} \mathrm{Mg}\left({ }^{3} \mathrm{He}, \mathrm{n}\right)^{26} \mathrm{Si}$ commissioning run in December, 2013. Shown are eight deuterated benzene scintillators and two HPGe detectors.

histograms related to these parameters were updated. The trigger rate was approximately $80 \mathrm{kHz}$, which resulted in a system live time of about $55 \%$. Further live time benchmarks showed system live time to be approximately $85 \%$ with a $15 \mathrm{kHz}$ trigger rate and system live time of $>99 \%$ for a $0.7 \mathrm{kHz}$ trigger rate. Compared to the nearly $90 \%$ dead time at a $3 \mathrm{kHz}$ trigger rate with the previous acquisition system at UKAL, the improvement is substantial. The data quality out of the sorting algorithm is excellent and subsequent, detailed analysis of the data set, as shown in Figure 9 , is well underway.

This material is based upon work supported by the U. S. National Science Foundation under Grant No. PHY-1305801 and the Department of Energy National Nuclear Security Administration under Award No. DE-NA0000979. 


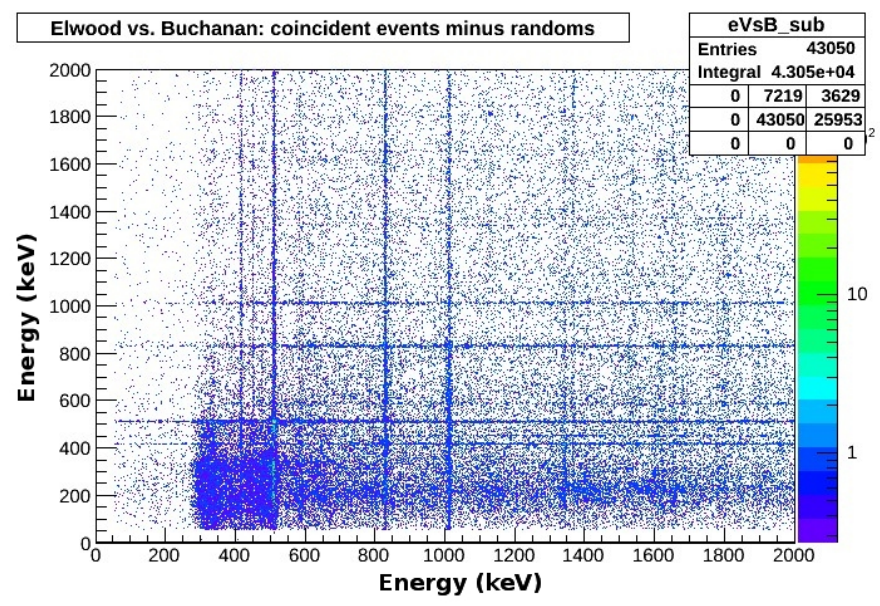

Figure 9: Energy vs. energy matrix from the two HPGe detectors used in the ${ }^{24} \mathrm{Mg}\left({ }^{3} \mathrm{He}, \mathrm{n}\right){ }^{26} \mathrm{Si}$ commissioning run performed in December, 2013. The image was made using ROOT [3].

\section{References}

[1] Sparrow Corporation. Sparrow - Advanced Tools for Industry, Education and Research. [Online]. Available: http://www.sparrowcorp.com/.

[2] W-IE-NE-R Plein \& Baus GmbH. WIENER, Plein \& Baus, Corp. - Plein \& Baus Electronics for Research and Industry. [Online]. Available: http://www.wiener-d.com/.

[3] Rene Brun and Fons Rademakers, ROOT - An Object Oriented Data Analysis Framework, Proceedings AIHENP'96 Workshop, Lausanne, Sep. 1996, Nucl. Inst. \& Meth. in Phys. Res. A 389 (1997) 81-86. See also http://root.cern.ch/.

[4] V. Bildstein, P.E. Garrett, J. Wong, S.F. Ashley, D. Bandyopadhyay, J. Bangay, L. Bianco, B. Hadinia, K.G. Leach, C. Sumithrarachchi, B.P. Crider, M.T. McEllistrem, E.E. Peters, S.W. Yates, and J.R. Vanhoy, et al., Comparison of Deuterated and Normal Scintillators for Fast-Neutron Detection, Nucl. Inst. \& Meth. in Phys. Res. A 729 (2013) 188-197. 